\title{
ARTICLE
}

\section{Beam power and residual dose history of J-PARC RCS}

\author{
Kazami Yamamoto*, Hideaki Hotchi, Hiroyuki Harada, Naoki Hayashi, Michikazu Kinsho, Pranab Kumar Saha, \\ Fumihiko Tamura, Masanobu Yamamoto, Masahiro Yoshimoto, Yoshihiro Nakane, Kazunari Seki \\ and Yukihiro Miyamoto \\ Japan Atomic Energy Agency, 2-4 Shirakata, Tokai-mura, Naka-gun, Ibaraki-ken, 319-1195, Japan
}

\begin{abstract}
A $3 \mathrm{GeV}$ rapid cycling synchrotron (RCS) at J-PARC was commissioned in October 2007. Afterwards, the beam intensity was increased through a beam study, and the RCS has continuously provided a proton beam $>100 \mathrm{~kW}$ to the neutron target since October 2009. With renewed efforts brought about by beam commissioning, we have reduced losses in the RCS and achieved low-loss operation. We present the history of the operational beam power and the residual dose distributions after operation.
\end{abstract}

\section{Keywords: high-energy accelerator; beam loss; beam power; dose distributions}

\section{Introduction}

The $3 \mathrm{GeV}$ rapid cycling synchrotron (RCS) at the Japan Proton Accelerator Research Complex (J-PARC) is a high-intensity proton synchrotron. It delivers an intense proton beam to the target for neutron production in the Materials and Life Science Experimental Facility (MLF) as well as to the Main Ring (MR) synchrotron at a repetition rate of $25 \mathrm{~Hz}[1]$. The RCS was commissioned in 2007 at an output beam power of 4 $\mathrm{kW}[2]$. Since 2008, the RCS has provided a $3 \mathrm{GeV}$ proton beam to the MLF and MR. We continued the beam commissioning effort to reduce losses in the RCS, and progress was made on increasing the output beam power. Just before the Great East Japan Earthquake in March 2011, the output beam power reached $>200$ $\mathrm{kW}$ [3]. The remainder of 2011 was spent in recovering from the damage done by the earthquake. Owing to the significant effort of all J-PARC members, we restarted beam commissioning in December 2011. The output beam power was not only immediately returned to its previous level but was also increased to nearly $300 \mathrm{~kW}$. This paper summarizes the history of the output beam power from the RCS and the residual dose levels under each condition.

\section{History of the output power and residual dose}

Figure 1 shows the typical dose distribution around the RCS accelerator component, and Figure $\mathbf{2}$ shows the history of the RCS output power and residual dose at major loss points from October 2007 to July 2012.

At an early stage, the beam power was only $4 \mathrm{~kW}$. We

*Corresponding author. Email: kazami@post.j-parc.jp studied the beam response with various parameters and found the operation tune that results in the least loss. We chose to operate with a betatron tune of $(6.42,6.42)$ [4], and this parameter enabled operation with a beam loss of $<1 \%$ even if the beam power was $>200 \mathrm{~kW}$.

Just before the earthquake, we had continuously provided a beam power of $>200 \mathrm{~kW}$ to the MLF for the previous three months. However, the earthquake destroyed many J-PARC facilities. A recovery operation that aimed to restart beam operation before the end of the year was planned and completed in a hurry. In the RCS case, the utility systems had suffered a lot of damage, but almost all the accelerator components were intact except the movement of magnets. Unfortunately, all magnet positions were shifted, and the maximum displacement was $10 \mathrm{~mm}$ horizontally and $5 \mathrm{~mm}$ longitudinally[5]. In spite of the alignment errors due to the earthquake, the simulation results indicated that the increment of loss was sufficiently small, and hence was acceptable[6]. Therefore, we decided to give user operation the highest priority; realignment is scheduled to be performed during the summer shutdown of 2013 . We carefully observed the beam loss monitor (BLM) signals and residual doses after operation, but we could not find a loss increment in the RCS with $200 \mathrm{~kW}$ continuous operation[7]. Moreover, we achieved user operation with nearly $300 \mathrm{~kW}$ output for 3 days. During this time, the total amount of loss was $<1 \%$, which is in good agreement with the simulation results.

Of course, even if only a $1 \%$ loss is dispersed in an accelerator, it can cause serious activation of accelerator components. However, because the RCS collimators perform sufficiently well, most losses were localized on the collimators[8]. Thus, only a small fraction was lost in components of the RCS other than the collimators. 


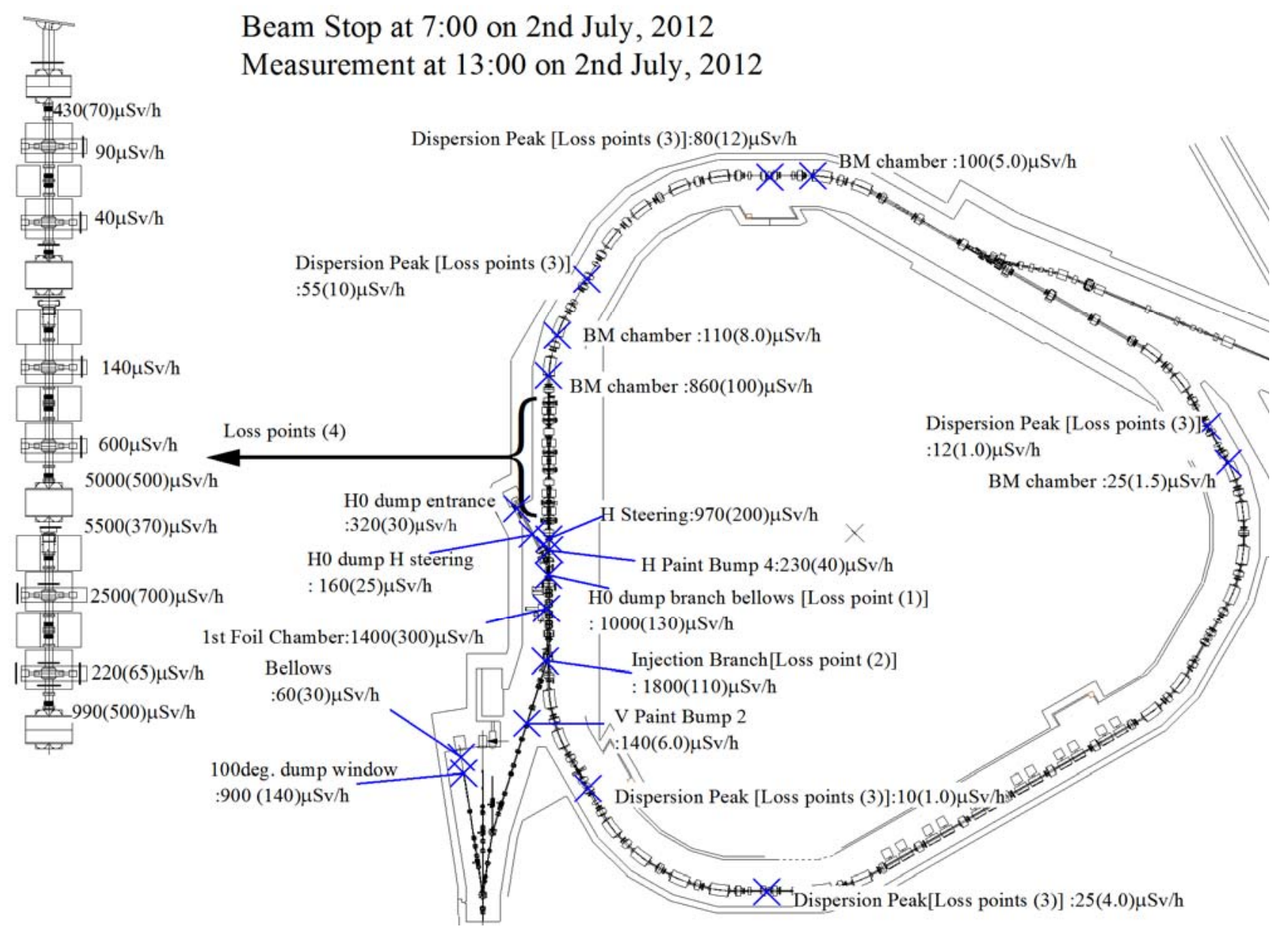

Figure 1. Residual dose distributions in the RCS after $200 \mathrm{~kW}$ operation for 1 month and $300 \mathrm{~kW}$ operation for 3 days. The values on the left are the residual doses on contact, and those on the right within parentheses are those at $30 \mathrm{~cm}$ away from the vacuum chamber surface.

From the measurement result of residual dose distribution, we can approximately say that there are four major loss points in the RCS: (1) at the branch duct of the circulating beam and the $\mathrm{H}^{0}$ waste dump, where we wasted an unexchanged component of the injection $\mathrm{H}^{-}$beam; (2) between injection septum magnets 1 and 2; (3) near the points of maximum dispersion; and (4) at the collimators. Among these, losses at the collimators were already considered. The collimator has a remote clamp system, and we can remove the collimator chamber from a great standoff distance[9,10]. We investigated the source of the other losses.

\section{Major loss points}

\subsection{Loss at the branch duct between the circulating beam and unexchanged waste beam}

The first significant loss was observed at the branch of the $\mathrm{H}^{0}$ dump line (black plot in Figure 2) when we started the RCS commissioning. Furthermore, there was another activation point at the beam position monitor (BPM) installed downstream of the $\mathrm{H}^{0}$ dump branch duct shortly after commencing user operation. The dose values of these points were $>1 \mathrm{mSv} / \mathrm{h}$ after 1 month at $120 \mathrm{~kW}$ output and $5 \mathrm{~h}$ of cooling.

In order to investigate the source of these losses, we conducted a beam study. The painting injection system is adapted to the RCS injection scheme; thus, we changed the painting pattern and measured the BLM signals in this study. The study result showed that the BLM response is proportional to the number of foil hits. This indicated that these losses were caused by the scattering of the injection and circulating beams at the charge exchange injection foil $[11,12]$.

We took three measures to mitigate these losses. First, we used painting injection, and second, we adjusted the foil position. These two measures minimized the number of foil hits. Third, we installed an additional collimator in the thick shielding wall at the branch in parallel with the recovery work from the earthquake. As a result, the loss caused by foil scattering decreased to $<20 \%[13,14]$. 


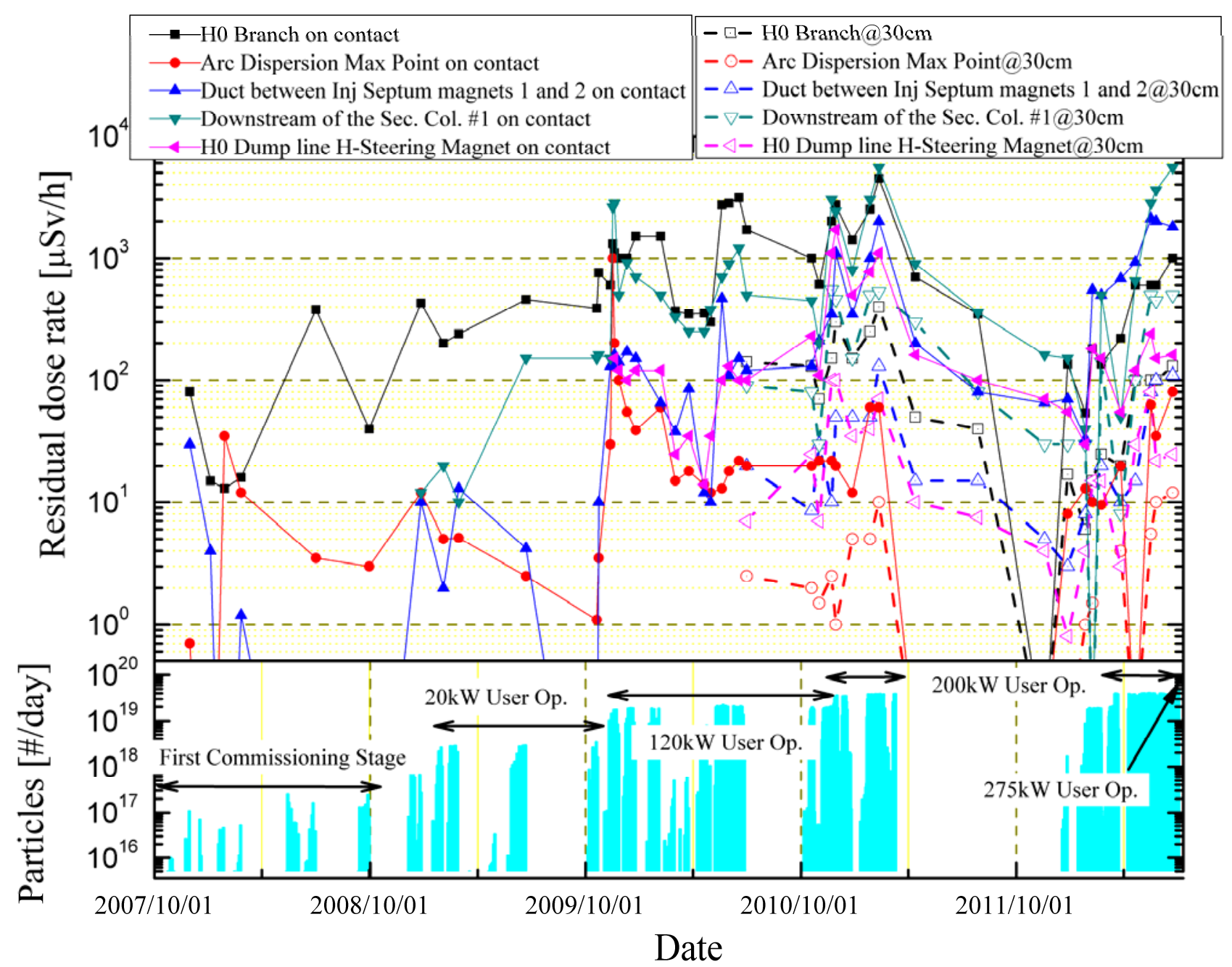

Figure 2. History of the RCS operation power and residual doses.

\subsection{Losses near the points of maximum dispersion}

There were few losses near the points in the arc of maximum dispersion. Usually the residual dose values were $<100 \mu \mathrm{Sv} / \mathrm{h}$ (red plot in Figure 2). The BLM signal indicated that these losses occurred during the middle of the acceleration period and were sensitive to tune variations, the excitation pattern of sextupole magnets, and RF patterns. We studied various operation patterns and chose the parameters that produced the least loss. Especially, we developed a multiharmonic feedforward compensation system. This system can make the amplitude and phase patterns that cancel the wake voltage during the acceleration period, thus we were able to keep large RF bucket and to reduce the arc loss[15].

When we started user operation with an output power of $>100 \mathrm{~kW}$, the dose values near the points of maximum dispersion increased temporarily. This occurred because the primary collimator was out of position. We immediately repositioned it, and the losses disappeared[16]. After that, the residual dose values remained at $<100 \mu \mathrm{Sv} / \mathrm{h}$, even at $300 \mathrm{~kW}$ user operation.

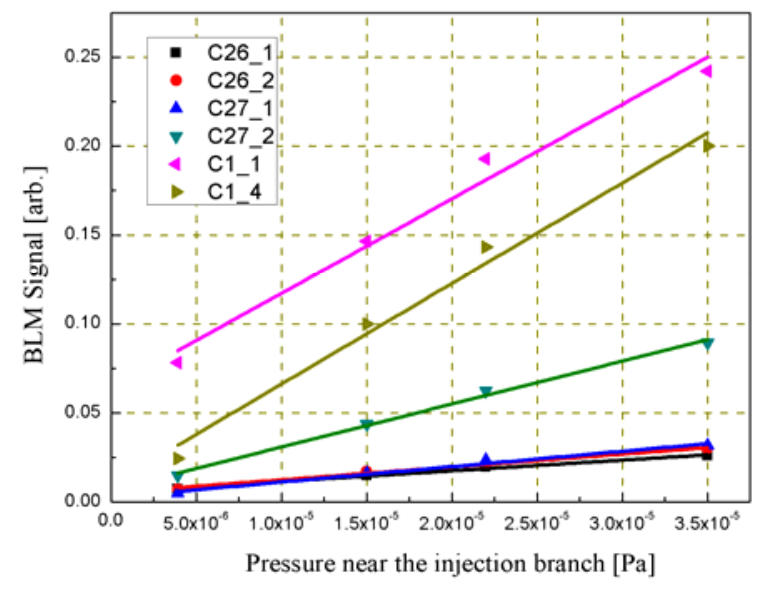

Figure 3. Dependence of beam loss on the pressure near the injection branch. 


\subsection{Loss between the injection septum 1 and 2}

Another loss was observed between injection septum magnets 1 and 2 at $120 \mathrm{~kW}$ user operation. In this case, the residual dose could be observed only at the opposite direction of the injection $\left(\mathrm{H}^{-}\right)$beam orbit of the vacuum chamber (blue plot in Figure 2). Therefore, we consider that the beam loss was probably caused by the charge-exchanged particles. Once, the pressure of the injection area was accidentally increased by the failure of a vacuum pump. At this point, the BLM signal also increased. Figure 3 shows the dependence of the BLM signal on pressure near the injection area. The BLM signal was in good proportion to the pressure. Thus, we will install additional pumps into the LINAC $-3 \mathrm{GeV}$ RCS beam transport (L3BT) line.

\section{Conclusion}

The RCS was carefully designed to minimize loss, and further, to localize loss on the collimator. To achieve design performance, the RCS beam study has been continued since the start of beam commissioning. Beam commissioning results indicated that the RCS established low-loss operation except at a few loss points. The loss caused by foil scattering was mitigated by using painting injection, adjusting the foil position, and installing an additional collimator. We found that the loss between injection septum magnets 1 and 2 is strongly dependent on the vacuum pressure; thus, we will improve the pressure along the injection line. As of now, the injection energy is $181 \mathrm{MeV}$, which is $<50 \%$ of that in the original design $(400 \mathrm{MeV})$. This means that the present space charge effect just after injection is larger than that of the original design. The amount of space charge tune shift is proportional to $\beta^{2} \gamma^{3}$, where $\beta$ and $\gamma$ are parameters in the relativity theory[17]. This indicates that the amount of space charge tune shift at a $300 \mathrm{~kW}$ output power with $181 \mathrm{MeV}$ injection energy is almost similar to that at a $1 \mathrm{MW}$ output power with 400 $\mathrm{MeV}$ (original) injection energy. Therefore, we confirmed the validity of the RCS design from the viewpoint of the space charge effect. The injection energy will be upgraded in summer 2013, which will then allow us to attempt higher beam power operation.

\section{References}

[1] Y. Yamazaki (edit.), Accelerator Technical Design Report for J-PARC, KEK-report 2002-13.

[2] K. Yamamoto, N. Hayashi, S. Hiroki, R. Saeki, T. Toyama, M. Teshima and K. Satoh, Radiation level in the J-PARC rapid cycling synchrotron after first study, Proceedings of EPAC'08, MOPC133, (2008).

[3] M. Kinsho, Status of J-PARC RCS, Proceedings of IPAC'12, THPPP083.

[4] H. Hotchi, H. Harada, N. Hayashi, M. Kinsho, P. K. Saha, Y. Shobuda, F. Tamura, K. Yamamoto, M. Yamamoto, M. Yoshimoto and Y. Irie, Beam loss reduction by a painting injection in the $3-\mathrm{GeV}$ rapid cycling synchrotron of the Japan Proton Accelerator Research Complex, Phys. Rev. ST-AB 15, 040402 (2012).

[5] N. Tani and H. Hotchi, Effect of J-PARC $3 \mathrm{GeV}$ RCS alignment after the Tohoku Earthquake in Japan, Proceedings of the 8th Annual Meeting of Particle Accelerator Society of Japan, MOPS164, Tsukuba, Aug. 1-3, 2011. [in Japanese]

[6] N. Tani and H. Hotchi, Study on the realignment plan for J-PARC $3 \mathrm{GeV}$ RCS after the Tohoku Earthquake in Japan, Proceedings of IPAC'12, WEPPP085.

[7] K. Yamamoto, N. Hayashi, H. Hotchi, P. K. Saha, H. Harada, M. Yoshimoto, M. Kinsho, R. Saeki, S. Hatakeyama and S. Katoh, Comparison of the residual doses before and after resumption of user operation in J-PARC RCS, Proceedings of IPAC'12, THPPP070.

[8] K. Yamamoto, The beam collimator system of J-PARC rapid cycling synchrotron, Proceedings of 42nd ICFA Advanced Beam Dynamics Workshop HB2008, (2008).

[9] K. Yamamoto and M. Kinsho, Development of the collimator system for the $3 \mathrm{GeV}$ rapid cycling synchrotron, Proceedings of the 2005 Particle Accelerator Conference (PAC05), Knoxville, (2005).

[10]K. Yamamoto, M. Okazaki, Y. Hirooka, Y. Takeuchi, A. Nakamura, H. Hanaue and M. Abe, Present status of beam collimation system of J-PARC RCS, Proceedings of the 10th Biennial European Particle Accelerator Conference (EPAC06), Edinburgh, (2006).

[11]P. K. Saha, Operational experience with J-PARC injection and extraction systems, Proceedings of the 46th ICFA Advanced Beam Dynamics Workshop HB2010, Morschach, Switzerland, TUO2B01 (2010).

[12]P. K. Saha, H. Hotchi, H. Harada, M. Yoshimoto, K. Yamamoto, Y. Yamazaki and I. Sugai, Systematic beam loss study due to the foil scattering at the $3-\mathrm{GeV}$ RCS of J-PARC, Proceedings of IPAC'10, THPEB018.

[13]K. Yamamoto, Y. Yamazaki, M. Yoshimoto, J. Kamiya, H. Harada, P. K. Saha, H. Hotchi, M. Kinsho and S. Kato, Foil scattering loss mitigation by the additional collimation system of J-PARC RCS, Proceedings of IPAC11, TUPS033.

[14] S. Kato, H. Harada, S. Hatakeyama, J. Kamiya, M. Kinsho, K. Yamamoto, Y. Yamazaki and M. Yoshimoto, Localization of the large angle foil scattering beam loss caused by multi-turn charge-exchange injection, Proceedings of IPAC12, MOPPD074.

[15] F. Tamura, M. Yamamoto, C. Ohmori, A. Schnase, M. Yoshii, M. Nomura, M. Toda, T. Shimada, K. Hara and K. Hasegawa, Multiharmonic rf feedforward system for beam loading compensation in wide-band cavities of a rapid 
cycling synchrotron, Phys. Rev. ST-A.B 14, 051004 (2011).

[16]K. Yamamoto, S. Kato, H. Harada, M. Yoshimoto, Y. Yamazaki, N. Hayashi, P. K. Saha, H. Hotchi, and M. Kinsho, Performance of the beam collimation system in the Japan Proton Accelerator Research Complex Rapid Cycling Synchrotron, to be published in Proceedings of ICRS12.

[17] L. J. Laslett, On Intensity Limitations Imposed by Transverse Space-charge Effects in Circular Particle Aceelerators, Proceedings of 1963 Summer Study on Storage Rings, BNL-7534, (1963) pp.324-367. 